\title{
Societal Value of Stem Cell Therapy in Stroke - A Modeling Study
}

\author{
Johanna Svensson ${ }^{a}$ Ola Ghatnekar ${ }^{a} \quad$ Arne Lindgren $^{b, f}$ Olle Lindvall ${ }^{b, c, e}$ \\ Bo Norrving ${ }^{b, c}$ Ulf Persson ${ }^{a}$ Zaal Kokaia ${ }^{b, d}$, e \\ ${ }^{a}$ The Swedish Institute for Health Economics (IHE), b Division of Neurology, Department of Clinical Sciences, Lund

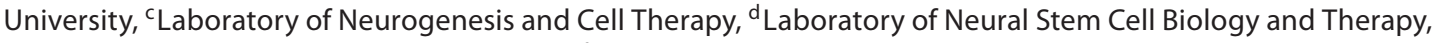 \\ eLund Stem Cell Center, Lund University, and fDepartment of Neurology, Skåne University Hospital, Lund, Sweden
}

\section{Key Words}

Stem cells $\cdot$ Cost effectiveness $\cdot$ Stroke

\begin{abstract}
Background: Stroke is one of the major causes of disability in the adult population and represents a heavy social and economic burden. Currently available therapeutic tools to support the recovery of impaired brain functions are quite limited. Animal studies have demonstrated that neuronal replacement and partial reconstruction of neural circuitry or modulation of the recovery process is possible with cell transplantation in the damaged adult brain. Stem cell therapy (SCT) may promote functional recovery also in stroke patients, thereby improving quality of life and reducing costs. Our aim was to estimate the potential societal value of SCT in stroke patients. Methods: We created a decision-analytic model in Microsoft Excel 2010 to assess life-long costs and quality-adjusted life years (QALYs) of SCT versus standard care for stroke patients from a societal perspective. The model structure consisted of 7 health states in accordance with the modified Rankin Scale (mRS). We modeled for age $(55,65$, and 75 years), functional status at discharge (mRS 2 , 3 , and 4), effectiveness of SCT (50 and $25 \%$ increase in the probability to improve $1 \mathrm{mRS}$ grade), mode of stem cell ad-
\end{abstract}

ministration, risk of recurrent stroke, complications of intervention, and use of immunosuppressive drugs. The difference between an assumed societal willingness to pay for a QALY gain in Sweden (110,400 USD) and the cost per QALY gain resulting from the model was interpreted as the value of SCT. Results: Increased survival (1.06 life years) and improved functional status gave rise to an estimated gain of 1.34 QALY in a cohort of patients aged 55 with mRS 2 at hospital discharge. Although the SCT intervention increased costs by 64,014 USD (excluding cost of stem cells), the costs of intervention were offset mainly by decreased productivity losses. In total, the intervention saved 19,055 USD, i.e., at a price of 19,055 USD for stem cells, the SCT would be cost neutral. The societal value of SCT was 166,500 USD. Conclusions: The application of the health-economic model to Sweden shows that in younger stroke patients with moderate disability, the societal value of SCT given a zero price of stem cells is 166,500 USD. Although the transplantation itself is more costly, SCT offers potential for cost offset and cost savings in a long-term perspective by reducing the disability after stroke. The therapy appeared cost effective under a wide range of assumptions. Hence, further research and development in stem cells suitable for stroke therapy could potentially produce great value to society.

Copyright $\odot 2012$ S. Karger AG, Basel

\section{KARGER \\ Fax +4161306 1234 \\ E-Mail karger@karger.ch}

www.karger.com
(C) 2012 S. Karger AG, Basel

$1015-9770 / 12 / 0336-0532 \$ 38.00 / 0$

Accessible online at:

www.karger.com/ced
Johanna Svensson

The Swedish Institute for Health Economics

Box 2127

SE-220 02 Lund (Sweden)

Tel. +46 463291 09, E-Mail js@ihe.se 


\section{Introduction}

Stroke represents the greatest cause of disability with about 46.6 million disability-adjusted life years (DALYs) [1]. The extension of the average human life span has resulted in a constant increase in the number of individuals with stroke among the expanding elderly population, creating a serious hazard to the quality of life and increased health care costs. According to WHO estimates, the number of stroke events in Europe is likely to increase from 1.1 million per year in 2000 to more than 1.5 million per year in 2025 solely because of the demographic changes [5]. In Sweden, the total excess direct and indirect cost of stroke is 1.8 billion USD [2]. The cost of stroke in Europe has been estimated to 53 billion USD per annum. Furthermore, on average, 39\% of total costs for stroke are attributable to morbidity and informal care giving costs [3]. In addition, there is an intangible cost to the patient for disability and relatives' reduced quality of life [4]. From this perspective, all efforts at bringing the latest advances of science and technology a step closer to the development of therapies against stroke should be vigorously promoted. Hence, if disability resulting from stroke could be ameliorated, and the post-stroke recovery could be improved, the gains would not be limited to reduced cost of care but also improved health-related quality of life. If more patients could return to work, the gain to society would be even greater in terms of reduced production losses.

New technologies for reducing morbidity among stroke patients are important in order to decrease the burden related to care. It is of great value to estimate the economic impact of new technologies early on in the development process. To estimate the impact of such technologies, explorative decision-analytic models are often used to simulate life-long effects not studied in clinical trials [5]. Even before clinical trials have measured the effectiveness of the new technology, modeling the economic impact based on an assumed effectiveness is useful. Results from early modeling studies can highlight where the value to society is expected to arise and stimulate further research aiming at clinical translation and development of commercially sound stem cell-based products for stroke therapy. However, results are expected to vary between countries due to different treatment practices, life expectancy, prices, and probability to improve or get a recurrent stroke resulting in differences in resource utilization.

Apart from thrombolysis during the first hours after an ischemic stroke, which can be given only to a limited proportion of patients, no effective pharmacological treatment to improve functional recovery exists in the post-ischemic phase. Several stem cell-based approaches are currently being explored in preclinical animal models of stroke [6], and guidelines for their clinical translation have been defined [7]. Neuronal precursors or neuroblasts derived from cultured stem cells are transplanted into the brain to restore damaged neural circuitry. Also, compounds are infused to stimulate neuron formation from the brain's own neural stem cells. Finally, and these approaches are closest to clinical application, stem cells are injected systemically and are believed to act through multiple mechanisms including, e.g., neuroprotection, modulation of inflammation, and stimulation of axonal plasticity, angiogenesis, and neurogenesis. Two initial clinical trials using stem cell therapy (SCT) approaches have been completed in patients with stroke [8-10], and several clinical trials with transplantation of bone marrow-derived stem cells as well as with a human fetal brain-derived cell line are ongoing or planned (www. clinicaltrials.gov). However, although a promising strategy for the functional restoration after stroke, SCT is still experimental.

The aim of this study was to estimate the value to society of SCT compared to standard care in stroke patients based on the incremental cost of SCT and the willingness to pay for a quality-adjusted life year (QALY) in Sweden. For this purpose, a health-economic model was designed to simulate costs, QALYs, and survival for stroke patients over a lifetime.

\section{Methods}

\section{The Model}

We created a decision-analytic model in Microsoft Excel 2010 to assess the value of SCT versus standard care among patients with ischemic stroke. We wanted to calculate costs and QALYs for both treatments, SCT and standard care, in a lifetime perspective. The model was designed to simulate treatment, functional development, and survival of the index event and long-term post-event management, respectively (fig. 1). The model was also developed to permit subgroup analyses according to age at stroke onset, functional status at hospital discharge, effectiveness of SCT, mode of stem cell administration, risk of recurrent stroke, and risk of death caused by intervention. The incremental cost-effectiveness ratio [ICER, defined as $\left(\right.$ Cost $_{\mathrm{SCT}}-$ Cost $\left._{\text {Standard }}\right) /\left(\right.$ Effect $_{\mathrm{SCT}}$ Effect $\left._{\text {Standard }}\right)$ ] describes the cost of producing an extra QALY with SCT compared to standard care, and can be used to inform decision makers on the potential value for money with SCT. Assuming a Swedish willingness to pay of 110,400 USD for a QALY gained $[11,12]$, the societal value of SCT can be expressed as the value of health benefits (here QALYs) less the incremental cost, i.e., 110,400 $\mathrm{USD}^{*} \mathrm{QALY}$ gain $-\left(\right.$ Cost $_{\mathrm{SCT}}-$ Cost $\left._{\text {Standard }}\right)$. Expressing the societal value in this way implicitly results in a cost-benefit analysis since both costs and benefits are expressed in monetary terms. 
Fig. 1. Decision tree describing the model. Cloned (i.e. identical) branches are not shown. Restroke $=$ Recurrent stroke.

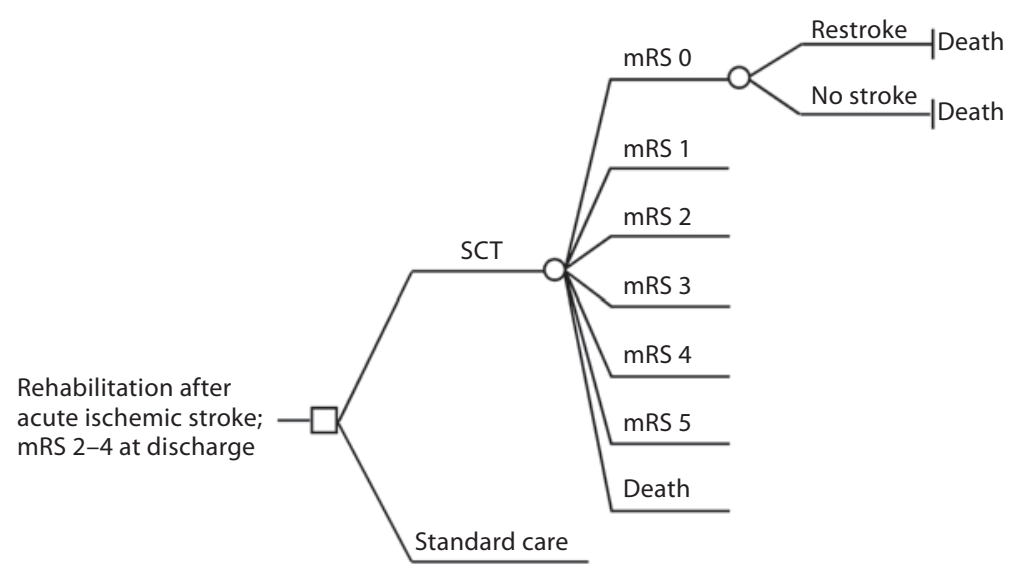

Table 1. Cohort mRS transition rates (patient flow) from hospital discharge to 3 months [15] score), the SCT affected survival indirectly. Two modes of administration of stem cells were considered: intracerebral through stereotaxic surgery and systemic via intravenous delivery. Intra-arterial delivery is also a possibility, but is not considered here.

As no clinical efficacy data were available for SCT, we relied on expert opinion for plausible assumptions on the size of the effects, which was set to a $50 \%$ increase in the chance to improve 1 score on the mRS scale. As a comparison, Goldstein et al. [13] showed in a prospective observational study on patients with ischemic stroke that approximately $26 \%$ more patients were discharged with little or no disability when they were cared for by a neurologist $(n=614)$ than if they were not $(n=161)$. Also, the NINDS trial showed that 39\% in the t-PA treatment arm had the outcome mRS 0-1 compared to $26 \%$ in the placebo arm [13].

\section{Post-Stroke Functional Development, Survival, and Quality} of Life

The model structure consisted of 7 health states in accordance with the mRS where 0 indicates no disability and mRS 6 is death. The mRS level at discharge from the hospital was used as the starting health state for the patients' post-stroke functional development. The transitions between health states for standard care treatment were governed by probabilities [14] during the first 3 months (table 1). After the first 3 months, we assumed that mRS steady state is reached and patients remain in the same mRS level until death unless they experienced a recurrent stroke. Recurrent strokes were modeled as deterioration in functional status by $1 \mathrm{mRS}$ unit at an annual risk of $4 \%$ [15]. We assumed that the disability level does not affect the risk of recurrent stroke. Quality of life weights for each $\mathrm{mRS}$ score were taken from the literature [16], ranging between 0.936 and -0.054 for mRS 0 and 5, respectively, with the latter indicating a situation worse than death. Apart from the first year, annual cycle length was used.
To: $\mathrm{mRS}$ at 3 months

mRS 0 mRS 1 mRS 2 mRS 3 mRS 4 mRS 5 Death

\begin{tabular}{lllllllll}
\hline \multicolumn{7}{c}{ To: $\mathrm{mRS}$ at 3 months } \\
\cline { 2 - 8 } & $\mathrm{mRS} 0$ & $\mathrm{mRS} 1$ & $\mathrm{mRS} 2$ & $\mathrm{mRS} 3$ & $\mathrm{mRS} 4$ & $\mathrm{mRS} 5$ & Death \\
\hline From: mRS & at hospital discharge \\
mRS 1 & 0.33 & 0.56 & 0.07 & 0.00 & 0.02 & 0.01 & 0.01 \\
mRS 2 & 0.07 & 0.54 & 0.20 & 0.11 & 0.04 & 0.00 & 0.05 \\
mRS 3 & 0.02 & 0.26 & 0.33 & 0.29 & 0.05 & 0.02 & 0.03 \\
mRS 4 & 0.02 & 0.10 & 0.13 & 0.30 & 0.32 & 0.04 & 0.09 \\
\hline
\end{tabular}

Survival was expected to vary by $\mathrm{mRS}$ and age at stroke onset. The survival in years 1-7 after stroke, stratified by mRS level, was taken from the work of Slot et al. [17], regarding survival in the Lothian stroke register in the UK. After year 7, we assumed a poststroke survival identical to that in the general Swedish population. SCT was not assumed to improve survival, but since life expectancy is increased with decreased mRS score, survival was indirectly affected by the experimental treatment. Survival was censored at 100 years. Survival for the first 7 years [17] was adjusted for age at stroke onset $(55,65$, and 75 years) by multiplying the survival with factors calculated from the literature [2] to generate age-specific survival by mRS level (table 2).

\section{Costs}

We adopted a societal perspective, i.e., including both direct and indirect costs expressed in USD in $2009^{1}$. Costs for secondary prevention and follow-up in primary care were not included because they were assumed identical for both treatment alternatives. All unit prices are presented in table 3.

First 3 Months. The resource use for the first 3 months was taken from a randomized, international multi-center study with

$1 \quad 1$ USD $(2009)=7.652$ SEK 
Table 2. Annual age cohort survival adjustment factors* in comparison to age 65 years

\begin{tabular}{llll}
\hline & \multicolumn{3}{l}{ Age at stroke onset } \\
\cline { 2 - 4 } & 55 years & 65 years & 75 years \\
\hline Time after stroke onset & & & \\
Year 1 & 1.01 & 1.00 & 0.98 \\
Year 2 & 1.03 & 1.00 & 0.94 \\
Year 3 & 1.06 & 1.00 & 0.87 \\
Year 4 & 1.10 & 1.00 & 0.81 \\
Year 5 & 1.13 & 1.00 & 0.79 \\
\hline
\end{tabular}

* A factor $>1$ indicates higher survival than a person aged 65 years.

Table 3. Swedish unit prices (2009)

\begin{tabular}{ll}
\hline & USD \\
\hline Health care and social services costs & \\
Hospital, inpatient stay per day at neurological clinic & 464 \\
Nursing home, short term per day & 201 \\
Home health care, per hour & 55 \\
Physiotherapy, per hour & 91 \\
Occupational therapy, per hour & 91 \\
Speech therapy, per hour & 91 \\
Nursing home, long-term annual cost/person & 67,074 \\
Home assistance, annual cost/person & 28,703 \\
Immunosuppressant therapy including & \\
$\quad$ administration, per month & 1,679 \\
MRI, per session & 412 \\
Intracerebral transplantation & 19,603 \\
Intravenous injection & 929 \\
\hline Productivity & \\
Mean monthly wage & 3,542 \\
Proportion of population, 16- to 64-year-old, in & \\
$\quad$ employment & $73.3 \%$ \\
Mean employees proportion of full time & \\
$\quad$ (2008, defined as 35 h/week) & $73.3 \%$ \\
Payroll taxes & $40 \%$ \\
\hline
\end{tabular}

820 patients with intracerebral hemorrhage [18]. The advantage with this source was that resources for length of hospitalization, home health care, and rehabilitation were stratified by the mRS score at 3 months after stroke. Swedish unit prices were applied to the resources.

Cost of Recurrent Stroke. The mean length of stay in hospital for a first-time stroke patient in Sweden was 22 days, whereas patients with recurrent stroke stayed 26 days [2]. To estimate the recurrent stroke cost, we inflated the initial hospitalization cost [18] with 26/22.

Value of Stem Cells in Stroke Therapy
Long-Term Costs - Social Services. No data were found in the literature for long-term costs after stroke based on mRS score. To estimate long-term costs, we assumed that mRS 0 and 1 incurred no long-term costs due to stroke, mRS 2 incurred $2 \mathrm{~h}$ of home health care per week, mRS 3 incurred full home health care, and that mRS 4 and 5 incurred the cost of living in a nursing home. These assumptions were guided by a study on the cost of stroke in Sweden [2]. If treatment affects survival, all costs (i.e., also nonrelated to stroke) for increased survival should be included. However, this principle has been debated and is not adopted in our model application [19].

Experimental Treatment. As stem cells suitable for stroke therapy are currently not commercially available, we set this cost to zero. In fact, even in direct contacts with companies having stem cell products in clinical trials, we could not obtain any cost estimation. Hence, the estimated societal value for SCT would indicate the maximum attainable cost for stem cells. Cost for an initial magnetic resonance imaging (MRI), immunosuppressant therapy, and administration of stem cells (intracerebral or systemic delivery) were added. The cost for immunosuppressive therapy refers to the regimen used in previous trials with fetal mesencephalic grafts in patients with Parkinson's disease, which included treatment with cyclosporine, azathioprine, and prednisolone with a mean duration of 29 months after transplantation [20]. Most investigators now agree that 12 months of treatment would be sufficient, and there may also be other modes of immunosuppression [21]. Costs were estimated for the doses indicated in the summary of product characteristics for each drug.

Productivity Losses. Indirect costs due to productivity losses were calculated for patients $<65$ years of age, the official retirement age in Sweden. Patients who died or had mRS score 3, 4, or 5 were assumed to exit the workforce. For patients $<65$ years of age with a milder stroke (mRS $0-2), 3$ months of sick leave were included before they returned to work. The value of productivity was calculated according to the human capital approach from the mean full-time employment wage of Sweden including pay roll taxes (factor 1.4). Adjustments for part-time employment and unemployment were made to represent the actual mean income of a person in the workforce.

\section{Analyses}

The base case scenario represented a patient cohort aged 55 years at stroke onset, with mRS 2 at hospital discharge, and an assumed increase in the probability to improve $1 \mathrm{mRS}$ grade of $50 \%$ with intracerebrally transplanted stem cells with no side effects. Both costs and effects were discounted by $3 \%$. In the univariate sensitivity analysis, some assumptions and variables were altered to study how sensitive the results were to these changes as an estimation of the robustness. The ranges in the sensitivity analyses were chosen by the authors using expert opinion.

\section{Results}

In the base case scenario, the estimated lifetime cost with the SCT procedure, excluding the cost of stem cells, was lower than with standard care, with cost savings of 19,055 USD (table 4). In the first 3 months after stroke 
Fig. 2. Estimated survival from standard care (SC) and SCT by age at stroke onset.

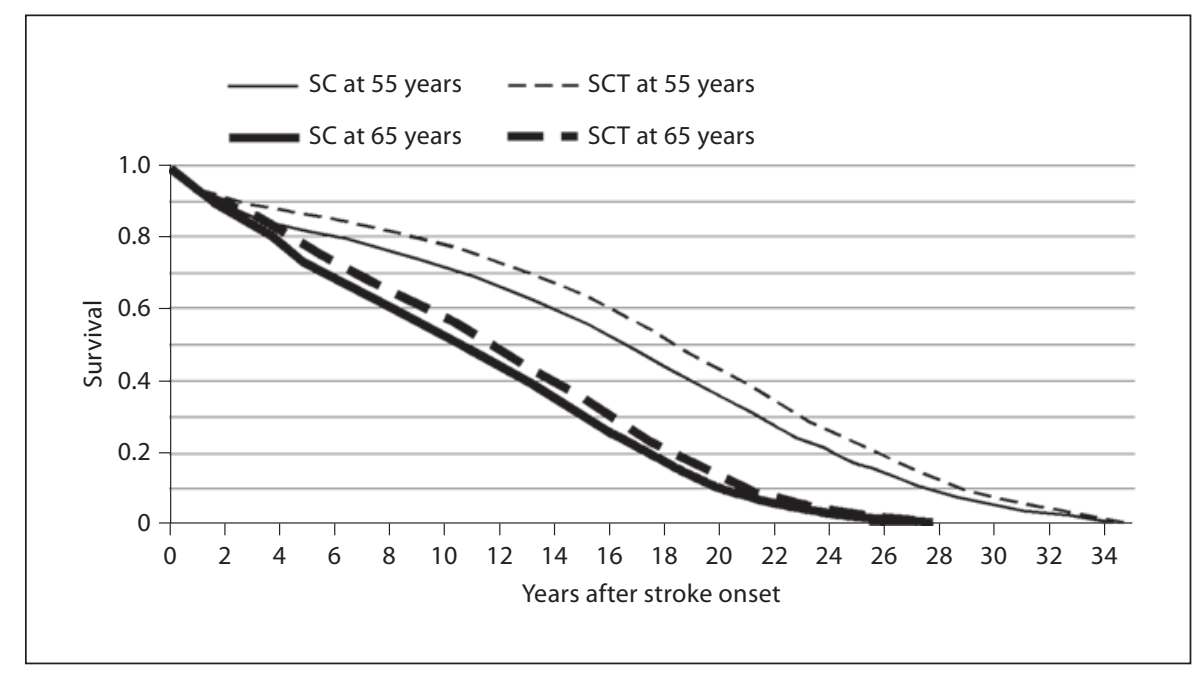

onset, the cost was higher for the SCT by 18,814 USD. However, some savings were realized as a result of patients moving to less severe and, hence, less costly health states. In the long-term perspective, the treatment cost was offset by savings in social services and indirect costs due to reduced mortality and disability levels. The cost for recurrent strokes did not differ in spite of the extended survival with SCT as the length of hospital stay was shorter for lower levels of disability. The estimated QALY gain was 1.34, primarily as a result of extended survival (1.06 life years) but also due to a shift to lower disability with higher quality of life. Figure 2 depicts the survival for two age cohorts (55 and 65 years) with both treatment alternatives. In the base case, the SCT generated a gain of 1 life year as patients moved to less disabling health states with lower mortality rates. Given the Swedish willingness to pay for a QALY, the societal value of SCT would be approximately 166,500 USD per treatment $[110,400$ USD $^{*} 1.34-(-19,055$ USD)]. In order for the intervention to be cost effective at the given willingness-to-pay threshold value, the effectiveness of the base case patient profile needs to be at least $14 \%$.

The sensitivity of the model was tested through oneway sensitivity analyses with the base case as the reference. The results are presented in table 5. Decreasing the assumption of a $50 \%$ relative efficacy of SCT from 50 to $25 \%$ decreased the QALY gain by $50 \%(1.34-0.67)$ while incremental costs increased to 22,000 USD. The mode of transplantation only affected the cost of the SCT procedure, not health outcomes compared to the base case. Likewise, a reduction in use of immunosuppressant medication to 12 months reduced the cost of SCT by an ad-
Table 4. Model results for SCT versus standard care under base case scenario (USD, 2009)

\begin{tabular}{|c|c|c|c|}
\hline & SCT & $\begin{array}{l}\text { Standard } \\
\text { care }\end{array}$ & Difference \\
\hline First 3 months, whereof & 56,863 & 38,049 & 18,814 \\
\hline Standard care & 7,063 & 8,522 & $-1,459$ \\
\hline Intracerebral transplant & & & \\
\hline and immunosuppression & 25,052 & 0 & 25,052 \\
\hline Rehabilitation & 3,368 & 4,234 & -867 \\
\hline Social services & 21,381 & 25,293 & $-3,912$ \\
\hline Long-term costs, whereof & 82,842 & 83,887 & $-1,045$ \\
\hline Immunosuppressants & 38,962 & 0 & 38,962 \\
\hline Recurrent stroke & 5,547 & 5,562 & -14 \\
\hline Social services & 38,333 & 78,325 & $-39,993$ \\
\hline Productivity losses & 63,196 & 100,020 & $-36,824$ \\
\hline Total & 202,901 & 221,956 & $-19,055$ \\
\hline Societal value of SCT & & & 166,525 \\
\hline Life years & 12.77 & 11.72 & 1.06 \\
\hline QALYs & 10.07 & 8.74 & 1.34 \\
\hline \multicolumn{3}{|c|}{ Incremental cost per life years gained } & SCT dominant \\
\hline \multicolumn{3}{|c|}{ Incremental cost per QALY gained } & SCT dominant \\
\hline
\end{tabular}

ditional 24,837 USD. Stroke onset at age 65 years reduced the QALY gain to 0.9 and potential cost offset for production losses and social services. Therefore, the societal value of SCT was lower for patients with stroke onset in older age than in the base case. Changes in the annual risk of recurrent stroke for both standard care and SCT affected the long-term costs as patients with recurrent stroke were assumed to worsen by $1 \mathrm{mRS}$ score and thereby needed more social services. However, because this re- 
Table 5. One-way sensitivity analyses of societal value of SCT versus standard care under different scenarios (USD, 2009)

\begin{tabular}{|c|c|c|c|c|}
\hline Analysis & Incremental cost & QALY gain & ICER & $\begin{array}{l}\text { Societal value of } \\
\text { SCT }\end{array}$ \\
\hline Base case scenario & $-19,055$ & 1.34 & SCT dominant & 166,525 \\
\hline $25 \%$ effectiveness & 22,178 & 0.67 & 33,214 & 51,558 \\
\hline Intravenous administration & $-37,729$ & 1.34 & SCT dominant & 185,199 \\
\hline Immunosuppression 12 months & $-43,892$ & 1.34 & SCT dominant & 191,362 \\
\hline Stroke onset age 65 years & 27,216 & 0.90 & 30,263 & 72,095 \\
\hline Stroke onset age 75 years & 29,557 & 0.54 & 54,579 & 30,246 \\
\hline Annual recurrent stroke risk 5\% & $-18,938$ & 1.29 & SCT dominant & 161,323 \\
\hline SCT procedure risk of death $5 \%$ & $-19,393$ & 1.32 & SCT dominant & 165,106 \\
\hline Intervention on $\mathrm{mRS} 3$ & $-14,891$ & 0.56 & SCT dominant & 76,978 \\
\hline Intervention on mRS 4 & 38,140 & 0.48 & 80,253 & 14,341 \\
\hline Sick leave 12 months & $-15,912$ & 1.34 & SCT dominant & 163,382 \\
\hline
\end{tabular}

The base case scenario represented a patient cohort aged 55 years at stroke onset, with mRS 2 at hospital discharge, and an assumed relative effectiveness of 50\% with intracerebrally transplanted stem cells with no side effects. Both costs and effects were discounted by $3 \%$.

sult was assumed for both treatment strategies, the resulting cost implications were small. The results were neither sensitive to a risk of death of $5 \%$ for the transplantation procedure [22], nor to an extended sick leave period up to 1 year after the index event. Intervention in patients with mRS 3 and 4 at hospital discharge in the acute phase reduced the scope for health benefits and cost offset. This occurred due to the fact that patients in mRS 3 and 4 had lower survival and gain in QALYs, as well as higher costs, than patients with mRS 2 at hospital discharge.

\section{Discussion}

Our study shows that in the base case, SCT generated both cost savings and health gains compared to standard care, resulting in a societal value of SCT up to 166,500 USD given a zero cost of the stem cells. This implies that the potential scope for cost recovery for investments in research aiming at developing stem cells for clinical use is substantial. For example, at a stem cell price of 19,055 USD per treatment, the intervention could be cost neutral, i.e., the SCT would bear its own cost from a societal perspective, and generate 1.34 extra QALYs. The results were sensitive to the assumed efficacy of SCT, age, and level of disability, but not very sensitive to the mode (or cost) of administration, the risk of recurrent stroke, and intervention-related mortality. The ICER increases by age mainly due to the inclusion of indirect costs. The young- er the patient is, the greater the savings in productivity losses.

The model application clearly showed the importance of the long-term perspective. Even a quite costly intervention can be justified since it was associated with benefits and saved costs for defined patient categories. Our study shows that the cost of intervention was not offset in the first year. Instead, the main potential for societal value was in the long-term benefits of reduced disability. The ability for patients to carry out usual activities not only influences the cost of care for the social services, but also the quality of life of patients and their relatives.

Ideally, health-economic analyses are based on data from long-term observational studies of broadly representative patient groups following general health care practices. For most treatments, however, only short-term data are available that do not cover all aspects of the disease. Disease modeling is a well-established method of generating long-term health-economic data in the absence of long-term empirical data. This information is important for decision makers, politicians, physicians, or researchers in their ex-ante decisions for health care technologies. Typically, mathematical simulation techniques are used to incorporate data from different sources into a cohesive epidemiological model. While the results must be interpreted carefully (e.g., due to uncertain parameters, expert opinions, or assumptions), health-economic models have a number of desirable features for decision making when complete information is not available. A 
review by Earnshaw et al. [5] included 13 modeling studies for acute stroke. The designs differed not only because of different settings but also because of different research questions. Our research question was to explore any potential societal gain with SCT, for different patient subgroups. We believe that deterministic one-way sensitivity analyses are illustrative to encourage research on subgroups where the potential societal value is the greatest. Once the technology is viable, probabilistic sensitivity analyses ought to be conducted to quantify the variation in the ICER due to the uncertainty to support health technology assessment and reimbursement decisions.

Several limitations of our study should be recognized. First, the effectiveness of SCT in humans has not yet been demonstrated and any estimate of effect size is uncertain. The societal value is highly influenced by the effect size estimate used: we modeled for a 50 and a $25 \%$ probability of improving $1 \mathrm{mRS}$ grade (both of which yielded a favorable societal value), but even the lower estimate may be too optimistic in the light of the large number of negative trials in the neuroprotective field. The treatment would be cost effective down to an effect size of $14 \%$, which may still be clinically relevant. Second, the optimum time point to administer stem cells after stroke in humans remains to be established. In experimental studies, both subacute and chronic administration are currently being evaluated. Recovery processes may be engaged early after stroke and be a target for intervention [23]. These endogenous mechanisms may enhance the effect of SCT but may also be promoted by the SCT. However, our model did not include differential costs on early versus late administration. Third, the indication for the use of immunosuppression after SCT is not established. We modeled for use and non-use of immunosuppression, taking only drug costs into account. Immunosuppressive therapy may potentially cause side effects that may be different in patients with acute stroke than in patients with, e.g., Parkinson's disease. This aspect was not included into our model because of lack of any background data on this issue. A common limitation when constructing decisionanalytic models is that the results ultimately rely on the quality of the data that are used for the calculations. Ideally, standard care data reflecting survival, treatment patterns, and transition probabilities for mRS should be collected from the same setting. There are only a few studies $[24,25]$ with detailed description of the time course of post-stroke functional recovery. We used a study [14] that we considered to comprise the most reliable data. Furthermore, we assumed that the disability steady state was attained after 3 months regardless of the functional sta- tus. Although this may not be the case, long-term costs and effects that had an impact on the results have been supported by Earnshaw et al. [5]. Likewise, the best available report on health care consumption with a split on $\mathrm{mRS}$ is based on data for hemorrhagic stroke [18]. Although hemorrhagic stroke patients require more resources in the acute phase than ischemic stroke patients, the effect of this is likely minimal since the mean length of stay in Sweden was estimated to 22 days, whereas it was 25.7 days in the study by Christensen and Morris [18]. This would translate into an additional 1,853 USD $[2,18]$. Furthermore, rehabilitation after the first 3 months was not included as we assumed it to be identical irrespective of the intervention.

A previous study on Parkinson's disease has illustrated the value of early health-economic modeling for the clinical application of a future cell replacement therapy [26]. Despite the caveats, our current results indicate that SCT may be a cost-effective therapy for stroke patients under a wide range of assumptions. The data from this study can support researchers to determine which patients should be targeted with SCT therapy and what cost items drive the results. More research is needed about the efficacy of SCT in order to estimate the costs and benefits with better precision. In addition, stem cells suitable for the therapy need to be developed and marketed. This study could therefore be considered as an attempt to estimate the potential value for further research in stroke therapy in order to make the best societal use of research investments [27].

\section{Conclusion}

The application of the health-economic model to Sweden shows that in younger stroke patients with moderate disability, the societal value of SCT given a zero price of stem cells is 166,500 USD. Although the transplantation itself is more costly, SCT offers potential for cost offset and cost savings in a long-term perspective by reducing the disability after stroke. Hence, further research and development in stem cells suitable for stroke therapy could potentially produce great value to society.

\section{Acknowledgements}

The study was funded by Lund University, EU project LSHB037526 (StemStroke) and 279017 (TargetBraIn), the Swedish Research Council, AFA Foundation, and the Swedish Government Initiative for Strategic Research Areas (StemTherapy). 


\section{References}

1 World Health Organization: The global burden of disease: 2004 update. http://www. who.int/healthinfo/global_burden_disease/2004_report_update/en/index.html (accessed January 27, 2012).

-2 Ghatnekar O, Persson U, Glader EL, Terént A: Cost of stroke in Sweden: an incidence estimate. Int J Technol Assess Health Care 2004;20:375-380.

3 Allender S, Scarborough P, Peto V, Rayner M, Leal J, Luengo-Fernandez R, Gray A: European cardiovascular disease statistics 2008. European Heart Network 2008.

4 Olai L: Life after a Stroke Event. With Special Reference to Aspects on Prognosis, Health and Municipality Care Utilization, and Life Satisfaction among Patients and Their Informal Caregivers. Dissertation at the Faculty of Medicine, Uppsala University, Uppsala, 2010.

$\checkmark 5$ Earnshaw SR, Wilson M, Mauskopf J, Joshi AV: Model-based cost-effectiveness analyses for the treatment of acute stroke events: a review and summary of challenges. Value Health 2009; 12:507-520.

$\checkmark 6$ Lindvall O, Kokaia Z: Stem cell research in stroke: how far from the clinic? Stroke 2011; 42:2369-2375.

7 Savitz SI, Chopp M, Deans R, Carmichael ST, Phinney D, Wechsler L: Stem cell therapy as an emerging paradigm for stroke (STEPS) II. Stroke 2011;42:825-829.

8 Bang OY, Lee JS, Lee PH, Lee G: Autologous mesenchymal stem cell transplantation in stroke patients. Ann Neurol 2005;57:874882.

>9 Kondziolka D, Steinberg GK, Wechsler L, Meltzer CC, Elder E, Gebel J, Decesare S, Jovin T, Zafonte R, Lebowitz J, Flickinger JC, Tong D, Marks MP, Jamieson C, Luu D, BellStephens T, Teraoka J: Neurotransplantation for patients with subcortical motor stroke: a phase 2 randomized trial. J Neurosurg 2005; 103:38-45.
10 Lee JS, Hong JM, Moon GJ, Lee PH, Ahn YH, Bang OY: A long-term follow-up study of intravenous autologous mesenchymal stem cell transplantation in patients with ischemic stroke. Stem Cells 2010;28:1099-1106.

11 Persson U, Hjelmgren J: Hälso-och sjukvården behöver kunskap om hur befolkningen värderar hälsan. Lakartidningen 2003;43:3436-3437.

12 Agency. TDaPB: Tlv: S uppdrag angående omregleringen av apoteksmarknaden. 2010: 50.

13 Goldstein LB, Matchar DB, Hoff-Lindquist J, Samsa GP, Horner RD: VA Stroke Study: neurologist care is associated with increased testing but improved outcomes. Neurology 2003;61:792-796

14 Ovbiagele B, Saver J: Day-90 acute ischemic stroke outcomes can be derived from early functional activity level. Cerebrovasc Dis 2009;29:50-56.

15 Hardie K, Hankey G, Jamrozik K, Broadhurst R, Anderson C: Ten-year risk of first recurrent stroke and disability after first-ever stroke in the Perth community stroke study. Stroke 2004;35:731-735.

16 Rivero-Arias O, Ouellet M, Gray A, Wolstenholme J, Rothwell P, Luengo-Fernandez R: Mapping the modified Rankin Scale (mRS) measurement into the generic EuroQol (EQ5D) health outcome. Med Decis Making 2010;30:341-354.

17 Slot K, Berge E, Dorman P, Lewis S, Dennis M, Sandercock P: Impact of functional status at six months on long term survival in patients with ischaemic stroke: prospective cohort studies. BMJ 2008;336:376-379.
8 Christensen M, Morris S: Association between disability measures and short-term health care costs following intracerebral hemorrhage. Neurocrit Care 2008;9:313318.

19 Liljas B, Karlsson GS, Stalhammar NO: On future non-medical costs in economic evaluations. Health Econ 2008; 17:579-591.

-20 Piccini P, Pavese N, Hagell P, Reimer J, Björklund A, Oertel W, Quinn N, Brooks D, Lindvall O: Factors affecting the clinical outcome after neural transplantation in Parkinson's disease. Brain 2005;128:2977-2986.

21 Lindvall O, Bjorklund A: Cell therapy in Parkinson's disease. NeuroRx 2004;1:382-393.

22 Lindvall O, Hagell P: Status of neural repair clinical trials in brain disease; in Selzer M, Clarke S, Cohen L, Duncun P, Gage F (eds): Textbook of Neural Repair and Rehabilitation Neural Repair and Plasticity. Cambridge, Cambridge University Press, 2006, vol 1, pp 615-631.

23 Marshall RS, Zarahn E, Alon L, Minzer B, Lazar RM, Krakauer JW: Early imaging correlates of subsequent motor recovery after stroke. Ann Neurol 2009;65:596-602.

24 Spieler J, Lanoe J, Amarenco P: Socioeconomic aspects of postacute care for patients with brain infarction in France. Cerebrovasc Dis 2002;13:132-141.

25 Lai S, Duncan P: Stroke recovery profile and the modified Rankin assessment. Neuroepidemiology 2001;20:26-30.

26 Hjelmgren J, Ghatnekar O, Reimer J, Grabowski M, Lindvall O, Persson U, Hagell P: Estimating the value of novel interventions for Parkinson's disease: an early decision-making model with application to dopamine cell replacement. Parkinsonism Relat Disord 2006;12:443-452.

27 Johnston SC: The 2008 William M. Feinberg lecture: prioritizing stroke research. Stroke 2008;39:3431-3436 